\title{
Computer Study of the Physical Properties of a Copper Film on a Heated Graphene Surface
}

\author{
A. E. Galashev ${ }^{a}$ * and V. A. Polukhin ${ }^{b}$ \\ ${ }^{a}$ Institute of Industrial Ecology, Ural Branch of the Russian Academy of Sciences, \\ ul. Sofii Kovalevskoi 20, Yekaterinburg, 620219 Russia \\ *e-mail: galashev@ecko.uran.ru \\ ${ }^{b}$ Institute of Material Studies and Metallurgy, Ural Federal University named after the First President of Russia B. N. Yeltsin \\ (Ural State Technical University-UPI), ul. Mira 19, Yekaterinburg, 620002 Russia \\ Received February 8, 2013; in final form March 4, 2013
}

\begin{abstract}
The structural, kinetic, and mechanical properties of a copper film deposited on single-layer and two-layer graphenes have been studied in a molecular-dynamics model in the temperature range $300 \mathrm{~K} \leq T \leq$ $3300 \mathrm{~K}$. The film sizes are reduced in the "zigzag" direction more slowly than in the "armchair" direction. The differences have been found to appear in the behavior of copper atoms on single-layer and two-layer graphenes with increasing temperature. Copper atoms on the two-layer graphene have higher horizontal mobility over entire temperature range. However, $\mathrm{Cu}$ atoms on the single-layer graphene become more mobile in the vertical direction beginning from a temperature of $\sim 1500 \mathrm{~K}$. The stress tensor components of the copper film characterizing the action of forces on the horizontal areas have a sharp extremum at $T=$ $1800 \mathrm{~K}$ in the case of the single-layer graphene and are characterized by quite smooth behavior in the case of the two-layer graphene.
\end{abstract}

DOI: $10.1134 / \mathrm{S} 1063783413080118$

\section{INTRODUCTION}

The study of the interaction of copper with graphene is of interest due to circumstances related to the production and application of graphene. The methods of production of graphene can be divided into three main groups. The mechanical methods using the mechanical peeling belong to the first group. The second group includes chemical methods, and the third group includes epitaxial methods and the method of thermal decomposition of a $\mathrm{SiC}$ substrate. In the method of chemical gas cooling, graphene is synthesized by decomposition of a carbon-containing gas (e.g., methane) into carbon and hydrogen at a temperature about $1300 \mathrm{~K}$ in the presence of a metallic foil. Further cooling of the metallic substrate initiates pressing-out of diffused carbon atoms from the metal and the formation of the graphene film on the substrate surface. The advantages of a copper substrate as compared to, e.g., a nickel substrate are that the formation of graphene on copper occurs without carbon diffusion to the metal bulk, i.e., it takes place immediately on the copper foil surface [1]. This makes it easy to produce single-layer graphene. The graphene layer grown on a copper-foil substrate can be introduced into transparence electrodes of sensor displays [2]. First, a two-dimensional carbon crystal is separated from the metal using a polymer adhesive and then transported on a substrate of a sensor polyethylene terephthalate display. The application of a copper- graphene composite is thought to be promising for fabricating a self-cooling system of transistors, which cardinally solves the problem of cooling computer devices [3]. The copper-graphene composite prepared by electrochemical deposition of copper on graphene nanoplates has a thermal conductivity of $460 \mathrm{~W} /(\mathrm{m} \mathrm{K})$, while that of cooper is $380 \mathrm{~W} /(\mathrm{m} \mathrm{K})$. The application of this material in electronic systems with high heat generation provides the economy of resources due to a smaller expenditure of cooper. Deposition of copper on graphene is of significant interest in relation to producing nanomaterials with unusual magnetic properties [4]. The magnetism of complexes formed by $\mathrm{Cu}$ atoms appears from $s$ - and $p$-states (to $30 \%$ ) and owing to surroundings of $\mathrm{Cu}$ atoms with $\mathrm{C}$ atoms. Point defects influence the magnetism of copper-carbon complexes. The mechanism of formation of defects in graphene at high temperatures can be studied in a computer experiment. Such a simulation also makes it possible to observe the behavior of the metallic atoms introduced into defects.

The aim of this work is to study the changes in the mechanical and kinetic characteristics of a singlelayer copper film adsorbed on single-layer and twolayer graphenes with increasing temperature.

\section{COMPUTER MODEL}

The Tersoff potential used to describe interatomic interactions in graphene is based on the concept of the 
bond order. The potential energy between two neighboring $i$ th and $j$ th atoms is written [5] as

$$
\begin{aligned}
& V_{i j}=f_{C}\left(r_{i j}\right)\left[A \exp \left(-\lambda^{(1)} r_{i j}\right)-B b_{i j} \exp \left(-\lambda^{(2)} r_{i j}\right)\right], \\
& f_{C}\left(r_{i j}\right)= \begin{cases}1, & r_{i j}<R^{(1)} \\
\frac{1}{2}+\frac{1}{2} \cos \left[\pi\left(r_{i j}-R^{(1)}\right) /\left(R^{(2)}-R^{(1)}\right)\right], \\
R^{(1)}<r_{i j}<R^{(2)} \\
0, & r_{i j}>R^{(2)},\end{cases}
\end{aligned}
$$

where $b_{i j}$ is the multi-particle bond-order parameter describing in what manner the bond-formation energy (attractive part $V_{i j}$ ) is created at local atomic arrangement because of the presence of neighboring atoms. The potential energy is a multi-particle function of atomic positions $i, j, k$ and is determined by parameters

$$
\begin{gathered}
b_{i j}=\left(1+\beta^{n} \xi_{i j}^{n_{i}}\right)^{-1 /(2 n)}, \\
\xi_{i j}=\sum_{k \neq i, j} f_{C}\left(r_{i j}\right) g\left(\theta_{i j k}\right), \\
g(\theta)=1+\frac{c^{2}}{d^{2}}-\frac{c^{2}}{\left[d^{2}+(h-\cos \theta)^{2}\right]},
\end{gathered}
$$

where $\xi$ is the effective coordination number, $g(\theta)$ is a function of the angle between $r_{i j}$ and $r_{i k}$ which stabilizes the tetrahedral structure. We used the Tersoff potential parameters for carbon from [5].

The Sutton-Chen potential was successfully used for simulating both bulk metals and metallic clusters [6]. The Sutton-Chen potential energy is written as

$$
U^{\mathrm{SC}}=\varepsilon\left[\frac{1}{2} \sum_{i} \sum_{j \neq i} V\left(r_{i j}\right)-c \sum_{i} \sqrt{\rho_{i}}\right],
$$

where

$$
V\left(r_{i j}\right)=\left(a / r_{i j}\right)^{n}, \quad \rho_{i}=\sum_{j \neq i}\left(a / r_{i j}\right)^{m},
$$

where $\varepsilon$ is a parameter having the dimensionality of energy; $c$ is the dimensionless parameter; $a$ is a parameter having the dimensionality of length that is commonly chosen to be the lattice parameter; and $m$ and $n$ are positive integers $(n>m)$. The power form of the contributions makes it possible to successfully joint the short-range interactions which are represented by an $N$-particle terms with the Van der Waals "tail' that determines long-range interaction. For copper, we used the Sutton-Chen potential parameters as follows: $m=6, n=9, \varepsilon=12.382 \mathrm{meV}$, and $c=39.432$ [6].
The copper-carbon interaction was described using the Morse potential:

$V\left(r_{i j}\right)=D_{0}\left(\exp \left[-2 \alpha\left(r_{i j}-r_{m}\right)\right]-2 \exp \left[-\alpha\left(r_{i j}-r_{m}\right)\right]\right)$.

The simulation was performed with the Morse potential parameters as follows: $D_{0}=87 \mathrm{meV}, \alpha=1.7 \AA^{-1}$, and $r_{m}=2.2 \AA$ [7].

In the initial state, $\mathrm{Cu}$ atoms were disposed above the centers of hexagonal cells formed by the $\mathrm{C}$ atoms; in this case, each of adjacent carbon cells was missed in both the longitudinal and the transverse directions. The arrangement is justified, because the nearest distance between graphene atoms is $1.42 \AA$, while similar characteristic of a copper crystal is $2.556 \AA$. As a result, we have a flat arrangement of $\mathrm{Cu}$ atoms corresponding to the stretched (111) plane of the fcc structure with minimal distance between copper atoms $6.507 \AA$. The $\mathrm{Cu}$ atoms were approached to the $\mathrm{C}$ atoms by the distance $2.243 \AA$ calculated by the density functional theory [8]. The equations of motion are solved numerically using the four-order Runge-Kutta method with a time step of $10^{-16} \mathrm{~s}$. For each temperature, the calculation time was $10^{6}$ time steps or 100 ps. The calculations were started at a temperature of $300 \mathrm{~K}$. After every $10^{6}$ time steps, the system temperature was increased by $500 \mathrm{~K}$, and the calculations were continued. The latter seventh calculation was performed at a temperature of $3300 \mathrm{~K}$. Identical calculations were performed for two-layer graphene. The graphene sheets were arranged according to the Bernal packing $(A B A B \ldots)$ exactly in the same manner as is the case in bulk graphite. The distance between the graphene sheets was taken to be the value predicted in the density functional approximation (3.347 $\AA$ ) [9]. The interaction between the $\mathrm{C}$ atoms belonging to layers $A$ and $B$ was considered based on the application of the Lenard-Jones potential with parameters $\sigma_{\mathrm{CC}}=$ $3.2963 \AA$ and $\varepsilon_{\mathrm{CC}}=0.0052 \mathrm{eV} \mathrm{[10].} \mathrm{A} \mathrm{graphene} \mathrm{sheet}$ containing 210 atoms was expanded in horizontal $x$ and $y$ directions due to applying the periodic boundary conditions. The action of the periodic boundary conditions was also applied to the metallic film. In the vertical $z$ direction, the boundary conditions were free. The total energy of the free single-sheet graphene calculated at $T=300 \mathrm{~K}$ is $-7.02 \mathrm{eV}$, which agrees with the quantum-mechanics calculation $(-6.98 \mathrm{eV})[11]$. At this temperature, the calculated energy of the copper-graphene interaction is $-1.4 \mathrm{eV}$. In the temperature range $300 \mathrm{~K} \leq T \leq 1300 \mathrm{~K}$, the heat capacity $c_{V}$ of the single-layer graphene calculated from the fluctuations of the kinetic energy increases from 19 to $28 \mathrm{~J} /(\mathrm{mol} \mathrm{K})$, which corresponds to the experimental values of this quantity $(23.74-26.80 \mathrm{~J} /(\mathrm{mol} \mathrm{K})[12]$. 
The self-diffusion coefficient was calculated using the correlation function of the copper atom velocity

$$
D=\frac{1}{3} \int_{0}^{\infty}\left(\frac{1}{N} \sum_{i=1}^{N} \mathbf{v}\left(t+t_{0}\right) \mathbf{v}\left(t_{0}\right)\right) d t,
$$

where the angular brackets denote the averaging over a set of the initial instants of time $t_{0}$, and $N$ is the number of copper atoms.

The stress in the place of the $i$ th atom in the metallic cluster is found as [6]

$$
\begin{gathered}
\sigma_{\alpha, \beta}(i)=\frac{\varepsilon}{2 a^{2} \Omega_{i}} \sum_{i \neq j}^{k}\left[-n\left(a / r_{i j}\right)^{n+2}\right. \\
\left.+c m\left(1 / \sqrt{\rho_{i}}+1 / \sqrt{\rho_{j}}\right)\left(a / r_{i j}\right)^{m+2}\right] r_{i j}^{\alpha} r_{i j}^{\beta},
\end{gathered}
$$

where the volume occupied by an individual atom $\Omega_{i}$ can be associated with the volume of the Voronoi polyhedron related to atom $i$.

\section{CALCULATION RESULTS}

The initial arrangement of $\mathrm{Cu}$ atoms on the graphene sheet is optimal from the standpoint of their interaction with the substrate, but it is not equilibrium for a metallic system with a regular atomic packing at a temperature of $300 \mathrm{~K}$. Because of this, even at this temperature, the atoms significantly approach to each other, so that the metallic film size decreases, and this reduction is more substantial in the transverse ("armchair") direction as compared to the longitudinal ("zigzag") direction (Fig. 1). The determination of the film size is related to the determination of the average $x$ and $y$ coordinates of three edge nonevaporated $\mathrm{Cu}$ atoms. We consider atoms evaporated if they are spaced at more than $3 \AA$ from all other atoms. With increasing temperature, there are some oscillations in the size reduction in the "zigzag" direction; however, the film size almost monotonically decreases in the "armchair" direction. At any temperature, the size decrease in the "armchair" direction exceeds the decrease in the film length in the "zigzag" direction. At temperatures of $1300 \mathrm{~K}$ and higher, the film size decreases due to both approaching metallic atoms in the $x y$ plane and their evaporation (displacements in the $z$ direction). The longitudinal film size of two-layer graphene decreases more smoothly with increasing temperature, but the transverse size decreases not so fast as in the case of single-layer graphene. As a result, at $T=3300 \mathrm{~K}$, the length of the metallic film more significantly decreases in the "zigzag" direction for twolayer graphene and in the "armchair" direction for single-layer graphene. In [13] the molecular-dynamics model of two-dimensional hexagonal lattice using the Brenner potential [14] was used to study the melting of a graphene nanoribbon. The removal of atoms from the ribbon continuing with increasing the calcu-

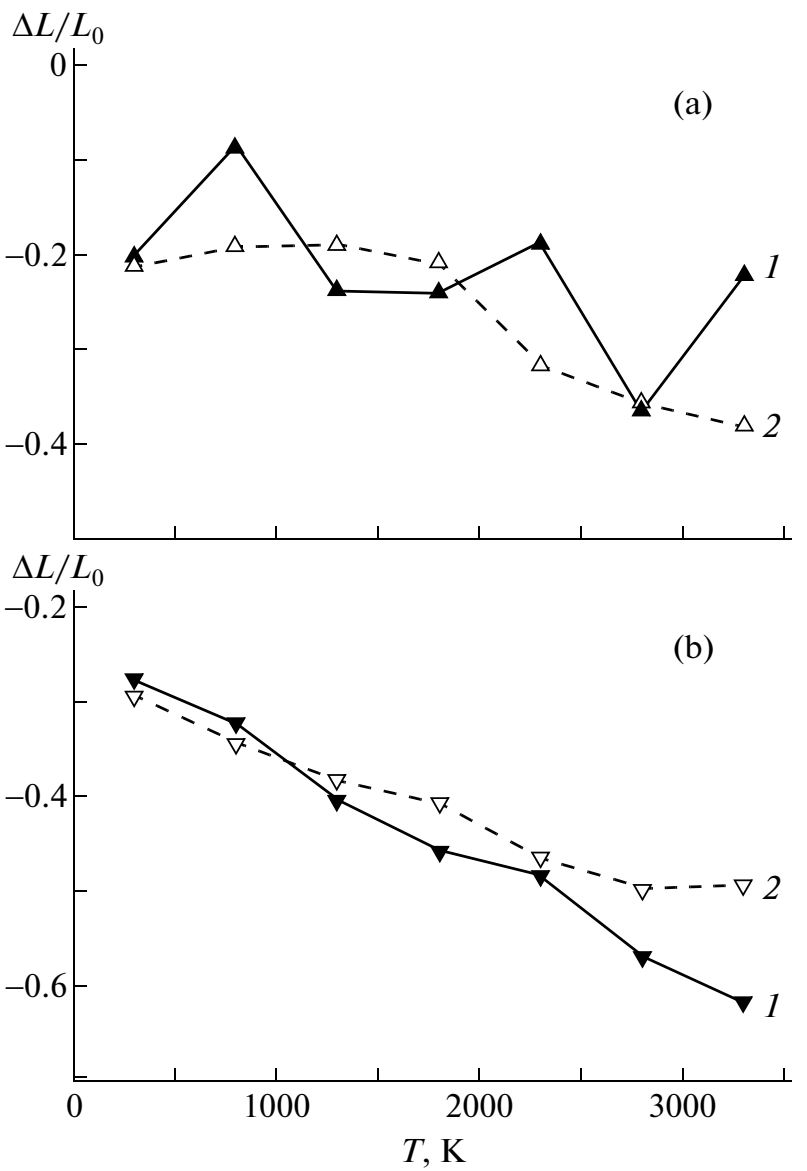

Fig. 1. Relative change in the copper film size in (a) the longitudinal and (b) transverse directions vs. temperature for the cases of (1) single-layer and (2) two-layer graphenes.

lation time and the formation of voids at $T=3400 \mathrm{~K}$ was interpreted as the melting of the graphene. However, the Monte Carlo method using the LCBOPII potential [16] and the two-dimensional Lindeman criterion gives a significantly higher $(4900 \mathrm{~K})$ melting temperature of graphene.

The transition of the metallic film to liquid state is accompanied by the concentration of majority of $\mathrm{Cu}$ atoms predominantly in the central region of the graphene sheet (Fig. 2). In this case, the main part of atoms is assembled as a flat droplet of rounded form. Such a pattern is observed at lower temperature $(1300 \mathrm{~K})$ as compared to the melting temperature of a bulk copper crystal $(1356 \mathrm{~K})$. In this state, two longitudinal cracks (from the center to the edges) form at the graphene sheet. At $T=1300 \mathrm{~K}$, the $\mathrm{Cu}$ atoms are no longer arranged in the $x y$ plane, but they have displacements in the $z$ direction (both up and down) which do not lead to their significant distances from the main material of the metallic film or graphene sheet. It is the state in which the penetration of a $\mathrm{Cu}$ atom to another side of the graphene sheet is observed 

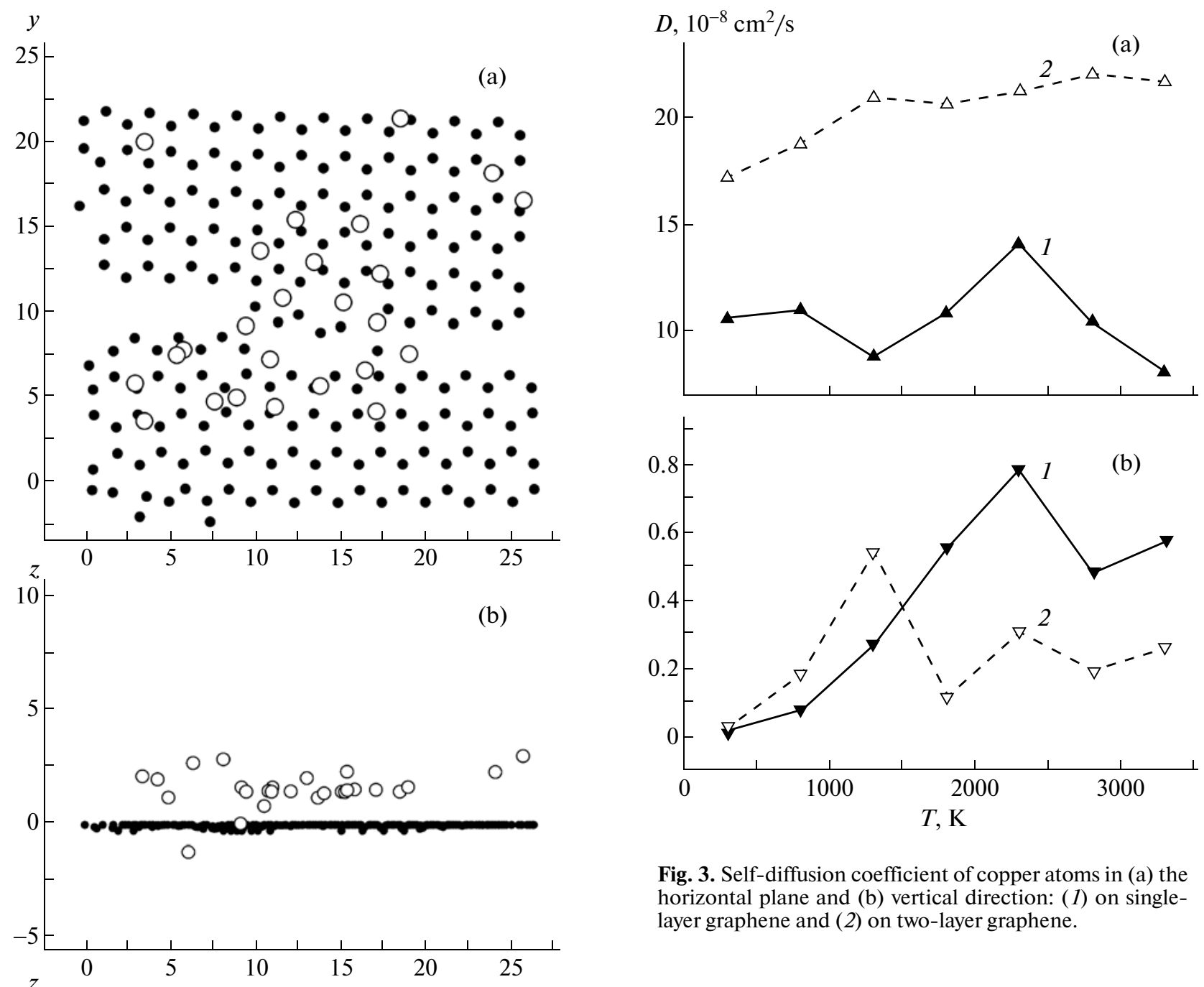

Fig. 3. Self-diffusion coefficient of copper atoms in (a) the horizontal plane and (b) vertical direction: (1) on singlelayer graphene and (2) on two-layer graphene.

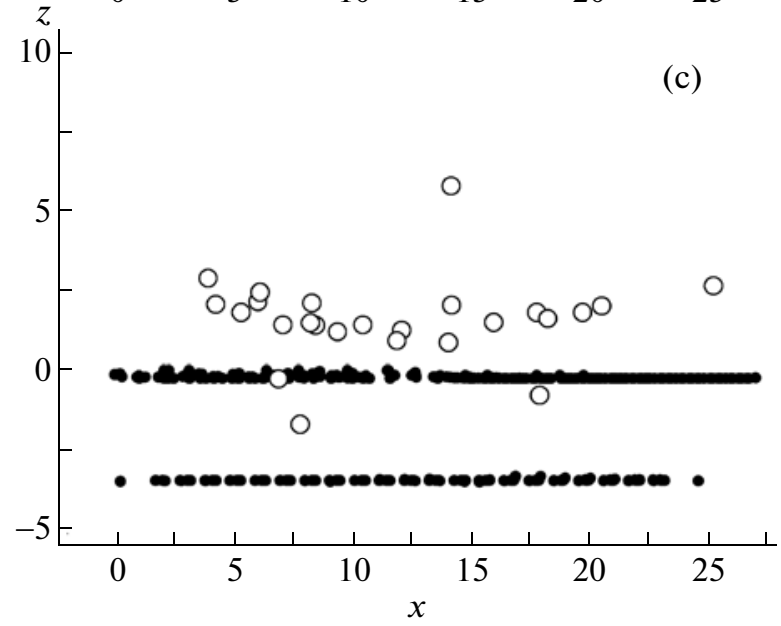

for the first time. At a temperature of $1800 \mathrm{~K}$, individual $\mathrm{Cu}$ atoms begin to evaporate up. The boiling temperature of massive liquid copper is more than $1000 \mathrm{~K}$ higher than this temperature $(2840 \mathrm{~K})$. The evaporation, along with the penetration of $\mathrm{Cu}$ atoms to another side of the graphene sheet, becomes markedly more intense at a temperature of $2300 \mathrm{~K}$. However, at $T=2800 \mathrm{~K}, \mathrm{Cu}$ atoms approach closely to each other and are forced against the graphene sheet, but at $T=$ $3300 \mathrm{~K}$, the evaporation and the penetration of $\mathrm{Cu}$ atoms to another side of the graphene sheet become more intense once again. The presence of another graphene layer changes the behavior of $\mathrm{Cu}$ atoms deposited on the first layer with increasing temperature. In this case, the cracks in the graphene sheet sufficient for $\mathrm{Cu}$ atoms to penetrate are formed even at $T$ $=800 \mathrm{~K}$. The atoms penetrated are attracted by the second graphene layer and remote from the first layer to larger distances than in the case of the single-layer graphene. However, in none of the cases, even at $T=$ $3300 \mathrm{~K}, \mathrm{Cu}$ atoms do not penetrate through the second graphene layer. More likely, the $\mathrm{Cu}$ atoms penetrating through the first graphene layer are merely 
accumulated in the gap between the graphene layers, because both the layers equally attract them.

The existence of the second graphene layer significantly increases the $\mathrm{Cu}$ atom mobility in the $x y$ plane and, at high temperatures, decreases it in the $z$ direction (Fig. 3). The mobility of $\mathrm{Cu}$ atoms in the vertical direction in the case of two-layer graphene is higher than in the case of single-layer graphene only to the temperature $T=1300 \mathrm{~K}$. In the case of single-layer graphene, the maximum of the self-diffusion coefficient $D$ is at a temperature of $2300 \mathrm{~K}$ in both the $x y$ displacements and the motion in the $z$ direction. For the two-layer graphene, the maximum of $D_{x y}$ corresponds to a temperature of $2800 \mathrm{~K}$, and the maximum of $D_{z}$ corresponds to a temperature of $1300 \mathrm{~K}$.

Because a temperature of $1300 \mathrm{~K}$ is close to the melting temperature of copper, we should expect the extrema in the dependence $D_{x y}(T)$. During collecting $\mathrm{Cu}$ atoms into a droplet on the single-level graphene, $D_{x y}$ first increased $(\mathrm{Cu}$ atoms were accelerated due to the forces of mutual attraction) and then decreased (at $T=1300 \mathrm{~K})$. The atomic mobility decreases due to the increase in the density of the "two-dimensional" liquid metallic droplet. Further increase in temperature leads once again to an increase in the horizontal mobility of atoms because of their motion beyond the $x$ and $y$ boundaries of the sheet and in the vertical direction. Up to $T=2300 \mathrm{~K}$, the larger contribution to the value of $D$ (for both directions) is that of atoms moving up and moving away from the sheet surface; another smaller part of atoms moves to the sheet surface. First, their contribution to the coefficient $D$ is relatively small, but with approaching temperatures higher than $2300 \mathrm{~K}$, they begin to significantly be slowed down by the carbon atoms meeting with them. As a result, coefficients $D_{x y}$ and $D_{z}$ decrease, and a main maximum appears in dependences 1 depicted in Figs. $3 \mathrm{a}$ and $3 \mathrm{~b}$. Coefficient $D_{x y}$ of $\mathrm{Cu}$ atoms disposed above the two-layer graphene has slightly observed maxima at $T=1300$ and $2800 \mathrm{~K}$. These extrema are also due to, on the one hand, approaching the atoms to each other and their collection into a liquid droplet which is damaged with increasing temperature and, on the other hand, the variation of the proportion of the contributions of the vertical atomic displacements in opposite directions. This variation is clearly observed in dependence 2 (Fig. 3b) that demonstrates an oscillating behavior with the maxima at 1300 and $2300 \mathrm{~K}$. The minimum observed in dependence $D_{z}(T)$ at $T=$ $2800 \mathrm{~K}$ (Fig. 3b) can be explained by the passage through the boiling point in the model followed by an increase in the $\mathrm{Cu}$ atom mobility with temperature.

The stress tensor components $\sigma_{z x}, \sigma_{z y}$, and $\sigma_{z z}$ characterizing the action of the coordinate components of the force in the $x y$ plane for the $\mathrm{Cu}$ film on the singlelayer and two-layer graphenes undergo substantially different changes with variations in the temperature (Fig. 4). In the case of single-layer graphene, the tem-

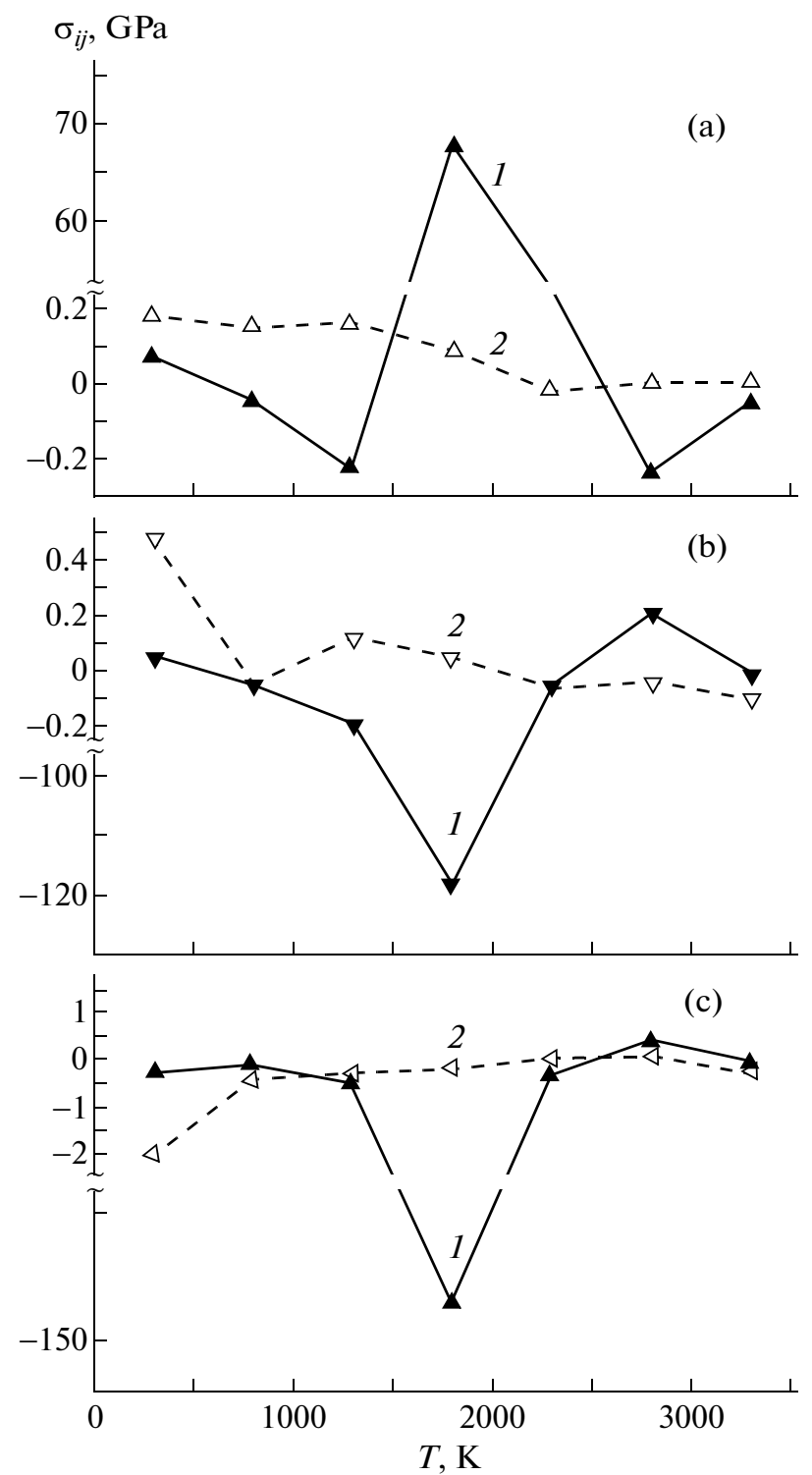

Fig. 4. Stress tensor components (a) $\sigma_{z x}$, (b) $\sigma_{z y}$, and (c) $\sigma_{z z}$ of the copper film deposited on (1) single-layer graphene and (2) two-layer graphene.

perature dependences of these parameters are complex. However, all the temperature dependences of the components have a general feature, namely, a sharp extremum at $T=1800 \mathrm{~K}$. At this temperature, the copper atoms form dense aggregates in the central part of the graphene sheet in places of disposing the bridges between cracks, i.e., the high stresses can be transmitted to the metallic film from the crack in the graphene sheet. At all other temperatures, these stress components for single and two-layer graphene have commensurate values. At the highest temperature $(T=3300 \mathrm{~K})$, the difference in the values of corresponding components (for the cases of single-layer and two-layer graphenes) is noticeably smaller than that at the lowest temperature $(T=300 \mathrm{~K})$. 


\section{CONCLUSIONS}

The copper atoms initially regularly arranged in interstitial sites of single-layer and two-layer graphenes form an instable metallic film which tends to decrease its size. The $\mathrm{Cu}$ film size decreases most significantly in the transverse ("armchair") direction in both single-layer and two-layer graphene. Heating of the film stimulates the decrease in its initial sizes. At high temperatures, the film is contracted due to approaching $\mathrm{Cu}$ atom to each other and due to the evaporation of $\mathrm{Cu}$ atoms from the substrate. In the "zigzag" direction, the temperature decrease in the film size is accompanied by significant fluctuations of the relative change in the length. As temperature increases, the longitudinal film size decreases more rapidly for copper deposited on two-layer graphene, and the transverse film size decreases more rapidly for copper deposited on single-layer graphene. Cracks formed on graphene during increasing temperature allow the penetration of the copper atoms to the opposite side of the sheet. In the case of two-layer graphene, the first $\mathrm{Cu}$ atom comes to the opposite side of the graphene sheet at a lower temperature $(800 \mathrm{~K})$. At high temperatures, the copper atoms penetrate through the upper sheet of the two-layer graphene more intensively than through the single-layer graphene. At all temperatures, the copper atom mobility in the horizontal direction is higher in the case of their deposition on the two-layer graphene; on the other hand, the mobility of copper atoms deposited on the single-layer graphene in the vertical direction becomes higher from a temperature of $\sim 1500 \mathrm{~K}$. The stress tensor components of the copper film reflecting the action of forces on the horizontal area behave differently in the cases of the film deposition on the single-layer or two-layer graphene. The very sharp extremum in the temperature dependences of their components in the case of depositing the film on the single-layer graphene appears at a temperature of $1800 \mathrm{~K}$ and can be due to the action of the fracture of the graphene sheet into "islands" of the dense packing copper atoms.

\section{REFERENCES}

1. M. G. Rybin, A. S. Pozharov, and E. D. Obraztsova, Phys. Status Solidi C 7, 2785 (2010).

2. P. A. Obraztsov, M. G. Rybin, A. V. Tyurnina, S. V. Garnov, E. D. Obraztsova, A. N. Obraztsov, and Y. P. Svirko, Nano Lett. 11, 1540 (2011).

3. K. Jagannadham, Metall. Mater. Trans. B 43, 316 (2011).

4. A. V. Krasheninnikov, P. O. Lehtinen, A. S. Foster, P. Pyykko, and R. M. Nieminen, Phys. Rev. Lett. 102, 126807 (2009).

5. J. Tersoff, Phys. Rev. Lett. 61, 2879 (1988).

6. H. Rafii-Tabar, Phys. Rep. 325, 239 (2000).

7. A. Oluwajobi and X. Chen, Int. J. Autom. Comput. 8, 326 (2011).

8. Z. Xu and M. J. Buehler, J. Phys.: Condens. Matter 22, $485301(2010)$

9. E. K. Yu, D. A. Stewart, and S. Tiwari, Phys. Rev. B: Condens. Matter 77, 195406 (2008).

10. M. C. F. Wander and K. L. Shuford, J. Phys. Chem. 114, 20539 (2010).

11. S. Yu. Davydov, Phys. Solid State 54 (4), 875 (2012).

12. G. K. White and S. J. Collocott, J. Phys. Chem. Ref. Data. 13, 1251 (1984).

13. J. Hu, X. Ruan, and Y. P. Chen, Nano Lett. 9, 2730 (2009).

14. D. W. Brenner, Phys. Rev. B: Condens. Matter 42, 9458 (1990).

15. K. V. Zakharchenko, A. Fasolino, J. H. Los, and M. I. Katsnelson, J. Phys.: Condens. Matter 23, 202202 (2011).

16. J. H. Los, L. M. Ghiringhelli, E. J. Meijer, and A. Fasolino, Phys. Rev. B: Condens. Matter 72, 214102 (2005).

Translated by Yu. Ryzhkov 